\title{
Substrate Distortion and the Catalytic Reaction Mechanism of 5-Carboxyvanillate Decarboxylase
}

\author{
Anna Vladimirova, ${ }^{\dagger}$ Yury Patskovsky, ${ }^{\ddagger}$ Alexander A. Fedorov, ${ }^{\ddagger}$ Jeffrey B. Bonanno, ${ }^{\ddagger}$ Elena V. Fedorov,
} Rafael Toro, ${ }^{\ddagger}$ Brandan Hillerich, ${ }^{\ddagger}$ Ronald D. Seidel, ${ }^{\ddagger}$ Nigel G. J. Richards, ${ }^{\S}$ Steven C. Almo, ${ }^{*}$, and Frank M. Raushel ${ }^{*} \dagger$

${ }^{\dagger}$ Department of Chemistry, Texas A\&M University, College Station, Texas 77843, United States

${ }^{\ddagger}$ Department of Biochemistry, Albert Einstein College of Medicine, 1300 Morris Park Avenue, Bronx, New York 10461, United States

${ }^{\S}$ Department of Chemistry and Chemical Biology, Indiana University-Purdue University Indianapolis, Indianapolis, Indiana 46202, United States

\section{Supporting Information}

ABSTRACT: 5-Carboxyvanillate decarboxylase (LigW) catalyzes the conversion of 5-carboxyvanillate to vanillate in the biochemical pathway for the degradation of lignin. This enzyme was shown to require $\mathrm{Mn}^{2+}$ for catalytic activity and the kinetic constants for the decarboxylation of 5-carboxyvanillate by the enzymes from Sphingomonas paucimobilis SYK-6 $\left(k_{\text {cat }}=2.2 \mathrm{~s}^{-1}\right.$ and $\left.k_{\text {cat }} / K_{\mathrm{m}}=4.0 \times 10^{4} \mathrm{M}^{-1} \mathrm{~s}^{-1}\right)$ and Novosphingobium aromaticivorans $\left(k_{\mathrm{cat}}=27 \mathrm{~s}^{-1}\right.$ and $k_{\mathrm{cat}} / K_{\mathrm{m}}=$ $\left.1.1 \times 10^{5} \mathrm{M}^{-1} \mathrm{~s}^{-1}\right)$ were determined. The three-dimensional structures of both enzymes were determined in the presence and absence of ligands bound in the active site. The structure of LigW from $N$. aromaticivorans, bound with the substrate analogue, 5-nitrovanillate $\left(K_{\mathrm{d}}=5.0 \mathrm{nM}\right)$, was determined to a resolution of $1.07 \AA$. The structure of this complex shows a remarkable enzyme-induced distortion of the nitro-substituent out of the plane of the phenyl ring by approximately $23^{\circ}$. A chemical reaction mechanism for the decarboxylation of 5-carboxyvanillate by LigW was proposed on the basis of the high resolution X-ray structures determined in the presence ligands bound in the active site, mutation of active site residues, and the magnitude of the product isotope effect determined in a mixture of $\mathrm{H}_{2} \mathrm{O}$ and $\mathrm{D}_{2} \mathrm{O}$. In the proposed reaction mechanism the enzyme facilitates the transfer of a proton to $\mathrm{C} 5$ of the substrate prior to the decarboxylation step.

\section{INTRODUCTION}

Microbial lignin degradation is of particular commercial and biotechnological interest, due to the ready availability of plant biomass for the production of renewable aromatic chemicals and biofuels. ${ }^{1}$ Lignocellulose is the primary structural material of terrestrial plant cell walls and is composed of the biopolymers cellulose, lignin, and hemicellulose. ${ }^{2}$ Lignin is an essential aromatic heteropolymer of phenylpropanoids produced by the oxidative polymerization of monolignols. ${ }^{3}$ The degradation of lignin is initiated by various oxidoreductases secreted by white rot fungi and further metabolized by specialized bacterial enzymes that are of importance for the terrestrial life cycle. However, the catalytic reaction mechanisms and three-dimensional structures for most of these enzymes remain to be elucidated, and there is a significant need for understanding and deciphering the microbial catabolic pathways that process oxidized lignin fragments. ${ }^{4}$

5-Carboxyvanillate decarboxylase (LigW) is a member of the amidohydrolase superfamily (AHS) and this enzyme catalyzes the nonoxidative $\mathrm{C}-\mathrm{C}$ bond cleavage of 5-carboxyvanillate (5$\mathrm{CV})$ to vanillate (VAN) as illustrated in Scheme 1a. The vast majority of the experimentally characterized enzymes from the AHS catalyze the hydrolytic cleavage of ester and amide bonds at carboxylate or phosphate centers contained within amino acids, sugars, and nucleic acids. ${ }^{5,6}$ However, LigW is associated with proteins from $\operatorname{cog} 2159$ and these enzymes catalyze decarboxylation and hydration reactions. Therefore, it is of considerable biochemical interest to understand how the active site of a superfamily of enzymes, that predominantly functions to catalyze the hydrolysis of phosphate and carboxylate esters, has evolved to catalyze the decarboxylation of substituted benzoic acids.

LigW may catalyze the decarboxylation of 5-CV using a variety of potential reaction mechanisms and examples of these transformations are illustrated in Scheme 2. The formation of vanillate requires the cleavage of the carbon-carboxylate bond at C5 of 5-CV and formation of a new carbon-hydrogen bond. The primary issues are whether $\mathrm{C}-\mathrm{H}$ bond formation precedes or follows the cleavage of the carbon-carboxylate bond, and

Received: August 14, 2015

Published: December 30, 2015 
Scheme 1

a

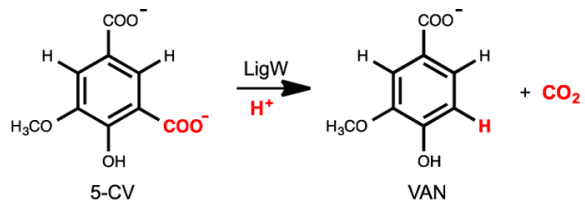

b<smiles>[O-]c1c([O-])c([O-])c([18O])c(O)c1[O-]</smiles><smiles>COc1cccc([O-])c1[N+](=O)[O-]</smiles>

c

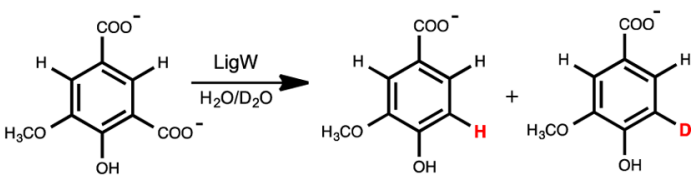

d

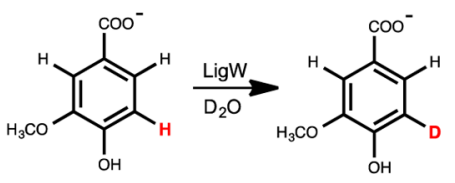

Scheme 2<smiles>O=C(O)c1c(O)c(O)c([18O])c(O)c1OCBr</smiles>

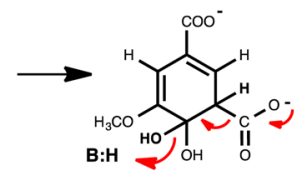

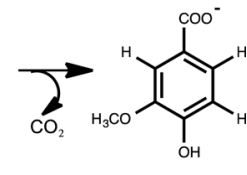

b<smiles>CCC(C)[C@H](CC)C(=O)O</smiles>

c
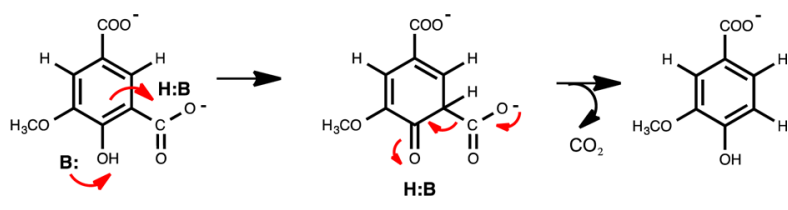

whether the initial reaction product is $\mathrm{CO}_{2}$ or $\mathrm{HCO}_{3}{ }^{-}$. Since $\mathrm{LigW}$ is a member of the AHS, this enzyme is homologous to a substantial number of enzymes that catalyze the hydrolysis of carboxylate esters and amides via the activation of a water molecule through coordination to a divalent cation within the active site. $^{5}$ In mechanism a, the reaction is initiated by the addition of water to the substrate. The hydrated intermediate subsequently collapses via cleavage of the carbon-carboxylate bond at $\mathrm{C} 5$ and the newly formed carbon-hydroxyl bond at $\mathrm{C} 4$ to generate the product VAN. In mechanism b, the C5carboxylate bond is broken to form $\mathrm{CO}_{2}$ and an unstable carbanion intermediate at $\mathrm{C} 5$ that is subsequently protonated by an active site acid. Alternatively, the lone pair of electrons from the ionized hydroxyl group at $\mathrm{C} 4$ can delocalize to the $\mathrm{C} 5$ carbon and be protonated. Decarboxylation of this intermediate leads directly to VAN (mechanism c). For each of these mechanisms alternative transformations can be envisioned by postulating the initial attack of water or an active site base on the C5-carboxylate group to ultimately result in the direct formation of bicarbonate rather than $\mathrm{CO}_{2}$ as the initial product. Here we propose a chemical reaction mechanism for the decarboxylation of 5-carboxyvanillate by LigW based on multiple high-resolution three-dimensional X-ray structures and the catalytic properties of this enzyme determined using heavy atom isotope effects and mutation of active site residues.

\section{RESULTS}

Catalytic and Molecular Properties of LigW. LigW from Spingomonas paucimobilis and Novosphingobium aromaticivorans was purified to homogeneity. The molecular mass of LigW from S. paucimobilis was determined to be $\sim 1090 \mathrm{kDa}$ by size exclusion chromatography, indicating that the enzyme (monomer $=38 \mathrm{kDa}$ ) associates in solution as a 32 -mer. When the gene for this enzyme was expressed in the presence of $1.0 \mathrm{mM}$ $\mathrm{MnCl}_{2}$, the purified enzyme contained 0.5 eq of $\mathrm{Mn}^{2+}$ per monomer. Dialysis of the $\mathrm{Mn}^{2+}$-substituted LigW against a solution containing $1.0 \mathrm{mM} \mathrm{MnCl}$, yielded protein with an average of $1.0 \mathrm{eq}$ of $\mathrm{Mn}^{2+}$ per subunit. The kinetic constants for the decarboxylation of 5-CV by LigW, prepared under these conditions, were obtained using assays supplemented with 1.0 $\mathrm{mM} \mathrm{MnCl}{ }_{2}\left(k_{\text {cat }}=2.2 \mathrm{~s}^{-1} ; K_{\mathrm{m}}=55 \mu \mathrm{M} ;\right.$ and $k_{\text {cat }} / K_{\mathrm{m}}=4.0 \times$ $\left.10^{4} \mathrm{M}^{-1} \mathrm{~s}^{-1}\right)$. The upper limit of $k_{\text {cat }}$ for the decarboxylation of 3-methoxy-5-carboxybenzoate (MCB, Scheme 1b) by LigW was less than $1.0 \times 10^{-3} \mathrm{~s}^{-1}$. The kinetic constants for the decarboxylation of 5-CV by LigW from S. paucimobilis were determined as a function of $\mathrm{pH}$ (Figure S1). For $k_{\text {cat }}$ the $\mathrm{pH}$ rate profile is bell-shaped with $\mathrm{p} K_{\mathrm{a}}$ and $\mathrm{p} K_{\mathrm{b}}$ values of $4.3 \pm 0.1$ and $8.3 \pm 0.1$, respectively. Similarly, for the $k_{\text {cat }} / K_{\mathrm{m}}$ profile the values of $\mathrm{p} K_{\mathrm{a}}$ and $\mathrm{p} K_{\mathrm{b}}$ are $4.7 \pm 0.2$ and $7.6 \pm 0.1$, respectively.

The gene for LigW from $N$. aromaticivorans was chemically synthesized and expressed in E. coli. The molecular mass of the purified enzyme determined by gel-filtration was $\sim 166 \mathrm{kDa}$. Since the molecular mass of an individual subunit of LigW from this organism is $40 \mathrm{kDa}$, this result is consistent with the formation of a tetramer. All catalytic activity was abolished after incubation of LigW from this organism with $o$-phenanthroline, thus confirming that this enzyme is also metal-dependent. The kinetic constants for decarboxylation of 5-CV catalyzed by LigW were measured at $\mathrm{pH} 7.1$ in the presence of $1.0 \mathrm{mM}$ of $\mathrm{MnCl}_{2}$ and are provided in Table S1.

Inhibition of Catalytic Activity. VAN, MCB, and 5nitrovanillate $(5-\mathrm{NV})$ were tested as inhibitors of the reaction catalyzed by LigW from S. paucimobilis. The nitro-analogue of the substrate (Scheme $1 \mathrm{~b}$ ) is a very potent inhibitor of LigW with an apparent inhibition constant, $K_{\mathrm{i}}^{\text {app }}$, of $17 \pm 7 \mathrm{nM}$ (Figure S2). The product vanillate and the analogue of the substrate that lacks the hydroxyl substituent at $\mathrm{C} 4$ (MCB) are much poorer inhibitors with apparent $K_{\mathrm{i}}$ values of $157 \pm 11 \mu \mathrm{M}$ and $960 \pm 92 \mu \mathrm{M}$, respectively. The dissociation constants for the LigW-inhibitor complexes, after correcting for the concentration of substrate used in the inhibition experiments, are $64 \pm 6 \mu \mathrm{M}, 390 \pm 60 \mu \mathrm{M}$, and $1.4 \pm 0.6 \mathrm{nM}$ for VAN, $\mathrm{MCB}$, and 5-NV, respectively. 5-NV is also a potent inhibitor of the reaction catalyzed by LigW from $N$. aromaticivorans with a $K_{\mathrm{i}}^{\mathrm{app}}$ of $17 \pm 4 \mathrm{nM}$ and a dissociation constant of $5.0 \pm 1.0 \mathrm{nM}$. The reversibility of the complex formed between LigW from $S$. paucimobilis and 5-NV was determined by mixing $88 \mu \mathrm{M} 5-\mathrm{NV}$ and $77 \mu \mathrm{M} \mathrm{LigW}$ at $\mathrm{pH}$ 7.0. In the absence of LigW, the solution of 5-NV is yellow with an absorbance maximum at 430 $\mathrm{nm}$ and an extinction coefficient of $4100 \mathrm{M}^{-1} \mathrm{~cm}^{-1}$. In the presence of LigW, the absorbance maximum increases to 470 $\mathrm{nm}$ and the extinction coefficient changes to $5210 \mathrm{M}^{-1} \mathrm{~cm}^{-1}$. If the $\mathrm{LigW} / 5-\mathrm{NV}$ complex is heated to $80{ }^{\circ} \mathrm{C}$ for $15 \mathrm{~min}$ to denature the protein, the visible absorbance spectrum of the 
filtrate is identical to that of 5-NV in the absence of added LigW.

Solvent Isotope Effects. The solvent isotope effects for the reaction catalyzed by LigW were determined at $\mathrm{pH}(\mathrm{D}) 7.0$ by direct comparison of the initial rates in $\mathrm{H}_{2} \mathrm{O}$ and $\mathrm{D}_{2} \mathrm{O}$. The measured solvent isotope effects on $k_{\text {cat }}$ and $k_{\text {cat }} / K_{\mathrm{m}}$ at $\mathrm{pH}(\mathrm{D})$ 7.0 are 2.9 and 2.0, respectively. The product isotope effect (PIE) was determined by measuring the ratio of deuterium to hydrogen in the product vanillate, when the decarboxylation of 5-CV by LigW was conducted in an equal mixture of $\mathrm{H}_{2} \mathrm{O}$ and $\mathrm{D}_{2} \mathrm{O}$ at a $\mathrm{pD} / \mathrm{pH}$ of 9.0 (Scheme 1c). The ratio of $\mathrm{H}: \mathrm{D}$ at $\mathrm{C5}$ was determined from the integrated signal intensity of the hydrogen at $\mathrm{C} 5$, relative to the nonexchangeable hydrogens at $\mathrm{C} 2$ and C6. From these measurements the PIE for the decarboxylation of 5-carboxyvanillate is 4.6.

Deuterium Exchange Experiments. The enzyme-catalyzed exchange of deuterium from solvent with the hydrogen at C5 of the product vanillate was determined by ${ }^{1} \mathrm{H}$ NMR spectroscopy (Scheme 1d). The initial reaction mixture contained $2.0 \mathrm{mM}$ vanillate and $65 \mu \mathrm{M} \mathrm{LigW}$ in $\mathrm{D}_{2} \mathrm{O}$ at $\mathrm{pD}$ 9.0. The solvent exchange reaction at $\mathrm{C} 5$ of vanillate resulted in the diminution of the NMR signal for the C5 hydrogen at 6.78 ppm and a loss of coupling to the C6 hydrogen at $7.33 \mathrm{ppm}$ (Figure 1). The observed first-order rate constant $\left(k_{\mathrm{obs}}\right)$ for the

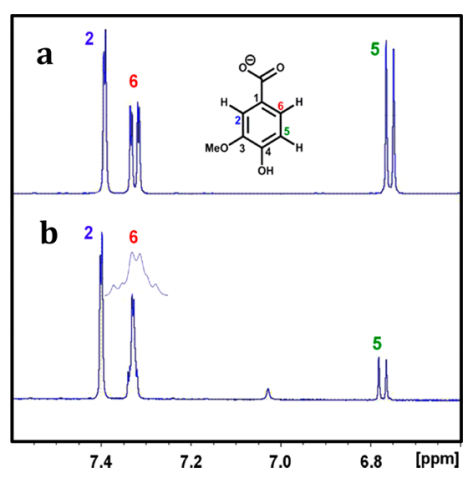

Figure 1. LigW-catalyzed exchange of deuterium at $\mathrm{C} 5$ of vanillate determined by ${ }^{1} \mathrm{H}$ NMR spectroscopy. ${ }^{1} \mathrm{H}$ NMR spectrum of vanillate in $\mathrm{D}_{2} \mathrm{O}$ at $\mathrm{pD} 9.0$ after incubating for 9 days at $25^{\circ} \mathrm{C}$ in the presence of $65 \mu \mathrm{M}$ LigW.

solvent exchange reaction at $\mathrm{pD} 9.0$ is $2.0 \pm 0.1 \times 10^{-4} \mathrm{~s}^{-1}$. The rate constant for the exchange reaction $\left(k_{\mathrm{ex}}\right)$, corrected for the concentration of enzyme and product used in this experiment, is $7.9 \times 10^{-3} \mathrm{~s}^{-1}$. No exchange $(<10 \%)$ was catalyzed by LigW with 3-methoxybenzoate after an incubation period of 7 days. No exchange $(<10 \%)$ was observed for control experiments with vanillate at $\mathrm{pD} 9.0$ in the absence of LigW after an incubation period of 7 days.

Three-Dimensional Structure of LigW from S. paucimobilis. The crystal structure of $\mathrm{LigW}$ with $\mathrm{Mn}^{2+}$ was determined to a resolution of $1.83 \AA$ (PDB id: 4ICM). Each protein subunit consists of a central distorted $(\beta / \alpha)_{8}$-barrel domain, where the active site and divalent cation are localized at the $\mathrm{C}$-terminal end of the $\beta$-barrel. An insertion domain is observed in this structure consisting of three helices $\alpha 1^{\prime}, \alpha 2^{\prime}$, and $\alpha 3^{\prime}$ (residues 23-70) between $\beta$-strand 1 and $\alpha$-helix 1 . Additional helices are found before and after $\alpha$-helix $5\left(\alpha 4^{\prime}\right.$ and $\left.\alpha 5^{\prime}\right)$ and after $\alpha$-helices $6\left(\alpha 6^{\prime}\right)$ and $8\left(\alpha 7^{\prime}\right)$. Two $3_{10}$ helices, $\eta 1^{\prime}$ and $\eta 2^{\prime}$, appear after $\beta$-strand 6 and $\alpha$-helix 7 , respectively (Figure S3). A single divalent cation is bound in the active site of LigW. The residues important for binding $\mathrm{Mn}^{2+}$ include Glu7 from $\beta$-strand 1, His-173 from $\beta$-strand 5, and Asp-296 from $\beta$-strand 8 . In addition to these three residues, there are three bound water molecules that complete an octahedral coordination geometry. Asp-296 from $\beta$-strand 8 also forms a hydrogenbonded network with His-226 and Glu-229. The structure of the metal binding site in LigW from S. paucimobilis is illustrated in Figure 2a.

Structures of LigW Inhibitor Complexes from S. paucimobilis. Crystal structures of LigW complexed with 5NV (PDB id: 4NG3), MCB (PDB id: 4NI8), and VAN (PBD id: 4L6D) were obtained at resolutions of $1.75,1.64$, and 1.45 $\AA$, respectively. In the structure of LigW with 5-NV, two of the metal-bound waters (Wat- 1 and Wat-2 of Figure 2a) are displaced by one of the oxygens from the nitro group at $\mathrm{C} 5$ and the hydroxyl group at $\mathrm{C} 4$, respectively. The octahedral geometry of the metal complex is retained (Figure $2 b$ ). The nitro-substituent further interacts with Arg-237 from the adjacent subunit. The carboxylate at $\mathrm{C} 1$ interacts electrostatically with Arg-45, and the methoxy group at C3 hydrogen bonds with the side chain hydroxyl from Thr-76. The phenyl group of the inhibitor is $\pi$-stacked with the side chain of Tyr299 at a distance of $3.7 \AA$. The structure of LigW complexed with $\mathrm{MCB}$ is identical to that of the complex with 5-NV except that the missing hydroxyl group from the inhibitor $\mathrm{MCB}$ is replaced by a water molecule that is coordinated to the divalent cation (Figure 2c). The rest of the molecular interactions with $\mathrm{MCB}$ are the same as found with 5-NV and VAN (Figure 2d). The crystal structures of LigW complexed with VAN, 5-NV, and MCB exhibit geometric distortion of the ligands when bound in the active site. For example, the hydroxyl group at $\mathrm{C} 4$ of VAN is distorted $\sim 15^{\circ}$ out of the plane of the aromatic ring (Figure S4a). The carboxylate group of $\mathrm{MCB}$ and the nitrosubstituent of 5-NV are also distorted from the plane of the aromatic ring with angles of $\sim 8^{\circ}$ and $11^{\circ}$, respectively (Figure $\mathrm{S} 4 \mathrm{~b}$ and Figure S4c). In addition, the angle between the hydroxyl group at $\mathrm{C} 4$ and the plane of the phenyl ring in the 5NV ligand is $\sim 22^{\circ}$ (Figure S4c).

Mutation of Active Site Residues. Ten residues localized in the active site of LigW from S. paucimobilis (Glu-7, Ala-9, Arg-45, His-173, Tyr-197, His-226, Glu-229, Arg-237, Asp-296, Tyr-299) were mutated and the purified proteins tested for changes in catalytic activity. Modification of any of the three residues that bind the divalent cation in the active site (Glu-7, His-173, or Asp-296) diminished the value of $k_{\text {cat }} / K_{\mathrm{m}}$ by at least three-orders of magnitude. His-226 and Glu-229 form an apparent hydrogen bonded network to the invariant aspartate (Asp-296) at the end of $\beta$-strand 8. Mutation of these residues to asparagine and glutamine, respectively, lowers the values of $k_{\text {cat }}$ and $k_{\text {cat }} / K_{\mathrm{m}}$ by $\sim 3$ orders of magnitude. The mutation of Ala-9 to histidine resulted in a relatively modest reduction in the catalytic activity of LigW. In most members of the AHS, this residue position is occupied by a histidine residue, which coordinates the active site metal ion. ${ }^{5}$ In the structure of LigW, Wat-3 is ligated to the metal ion at this location. Mutation of the tyrosine residues (Tyr-197 and Tyr-299) that interact with the phenyl ring of the substrate diminished the activity of LigW significantly. The two arginine residues (Arg-237 and Arg-45) that ion-pair with the two carboxylate groups of the substrate cannot be mutated to alanine without significant loss of catalytic activity. The kinetic constants for the decarboxylation of 5-CV by the LigW mutants are presented in Table S1. 

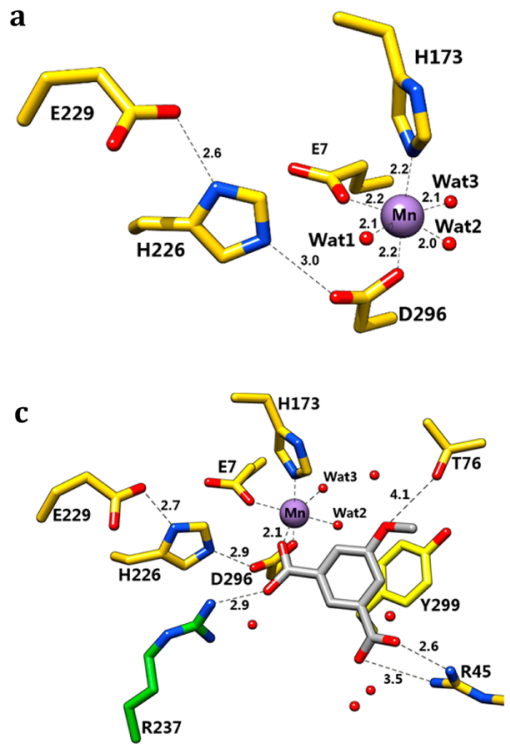
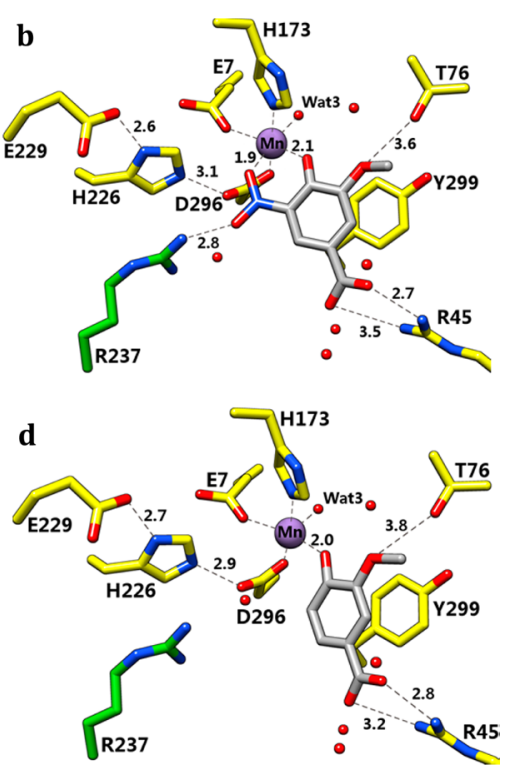

Figure 2. Active site architecture of LigW from S. paucimobilis in the presence of VAN, MCB, and 5-NV. (a) In the absence of added ligands, the manganese in the active site is coordinated to Glu-7, His-173, Asp-296 and three water molecules. (b) Active site structure of LigW in the presence of 5-NV. The inhibitor is presented with gray-colored bonds. (c) Active site structure of LigW in the presence of the MCB (presented in graycolored bonds). (d) Active site structure of LigW in the presence of the hydrolysis product, vanillate as shown in gray-colored bonds. In all panels manganese is shown as a purple-colored sphere. The arginine residue (Arg-237) from the adjacent subunit is colored green. The coordinate and hydrogen bonds are shown as black dotted lines with distances in Ångstroms.

Molecular Structure of LigW from N. aromaticivorans. The three-dimensional structure of wild-type LigW from $N$. aromaticivorans was determined in the presence and absence of 5-NV to resolutions of 1.07 and $1.47 \AA$, respectively (PDB ids: $4 \mathrm{QRN}$ and 4QTG). The minimal functional unit is a homodimer (Figure S5a), where each monomeric subunit folds as a distorted $(\beta / \alpha)_{8}$-barrel with an insertion domain of three helices between $\beta$-strand 1 and $\alpha$-helix 1 , which is important for substrate binding and specificity (Figure S5b). The active site is located at the dimer interface with an $\alpha$ helix of the opposite chain closing one side of the entrance to the active site. In the structure of LigW with 5-NV, two metalbound water molecules are displaced from the active site by the ligand (Figure 3a). Manganese coordinates 5-NV in a bidendate fashion via direct interactions with the hydroxyl group at $\mathrm{C} 4$ and the nitro-substituent at C5. The nitro group is also hydrogen-bonded to the side chains of His-241 from one subunit and Arg-252 from the adjacent subunit (Figure 3a). The carboxylate group at $\mathrm{C} 1$ of $5-\mathrm{NV}$ maintains strong polar contacts with the side chains of Tyr-51 (helix 2) and Arg-58 (helix 3). The phenyl ring of the ligand is sandwiched between Phe-212 (helix 10) and Tyr-317. The orientation of Tyr-317 is, in part, determined by a hydrogen bond between its phenolic group and the hydroxyl group of Thr-90. The overall structure of LigW appears relatively rigid. The arrangement of residues in and around the metal-binding site remains essentially the same in the presence and absence of 5-NV, except for the side chain of Asp-314 that adopts two alternate conformations when 5-NV is bound (Figure $3 a$ and $3 b$ ). It is noteworthy that one of the two conformations of Asp-314 places the side chain carboxylate group $3.1 \AA$ away from C5 of the ligand (Figure 3b), whereas an alternate conformation maintains the hydrogen bond between the same carboxyl oxygen and His-241 (2.9 A, Figure $3 b)$.

In the process of structure refinement, we observed that the geometry of 5-NV deviates significantly from the theoretically expected planar conformation. The nitro substituent of 5-NV is tilted out of a distorted aromatic ring plane (Figure 3c). The overall shape of the ligand reflects the shape of the binding pocket (Figure 3c). Since 5-NV and the substrate 5-CV have similar geometries, the overall distortion of the substrate is also likely to occur upon binding to the enzyme.

Geometric Distortion of 5-NV Upon Binding to LigW from $N$. aromaticivorans. A comparison of the structures for free and enzyme-bound 5-NV confirmed that geometric distortions of 5-NV occur only upon binding to LigW. The high resolution structure of free 5-NV is planar, with the hydroxyl and nitro substituents being in the same plane with the phenyl ring (Figure S6). By contrast, the planarity of 5-NV in the complex with LigW is distorted (Figure 4). The hydroxyl group and the $\mathrm{C} 3, \mathrm{C} 4$ and $\mathrm{C} 5$ carbons from the phenyl group all belong to one planar group (plane 1) that is tilted off the "main" ring plane $2(\mathrm{C} 1, \mathrm{C} 2, \mathrm{C} 6$, plus the carboxylate carbon) by approximately $13^{\circ}$. C5 and the nitro group make up another planar group (plane 3 ) that is about $23^{\circ}$ off of ring plane 2 .

Structure of the LigW D314N Mutant from $N$. aromaticivorans. The mutation of Asp-314 to asparagine causes more than a 1000 -fold reduction in the value of $k_{\text {cat }}$ relative to the wild-type enzyme (Table S1). Two structures of this mutant were determined; one with $\mathrm{Mn}^{2+}$ and 5-NV in the active site at a resolution of $1.80 \AA$ (PDB id: 4QS5) and one with only 5-NV at a resolution of $1.76 \AA$ (PDB id: 4QS6). The overall conformation of LigW is unaffected by the D314N mutation as the RMSD values between atomic coordinates of individual subunits of the structures did not exceed $0.3 \AA$. Moreover, the distorted conformation of 5-NV in the active site looks very similar in these structures. In the structure of D314N without a metal ion in the active site, a loosely coordinated water molecule replaces the manganese. The inhibitor 5-NV, however, is present in the active site and it adopts a similar distorted conformation as described for the other structures of LigW from N. aromaticivorans (Figure S7). 
a

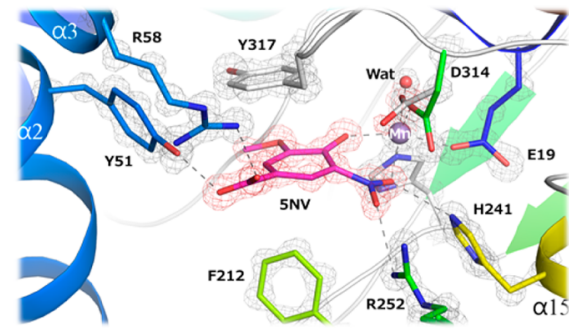

b
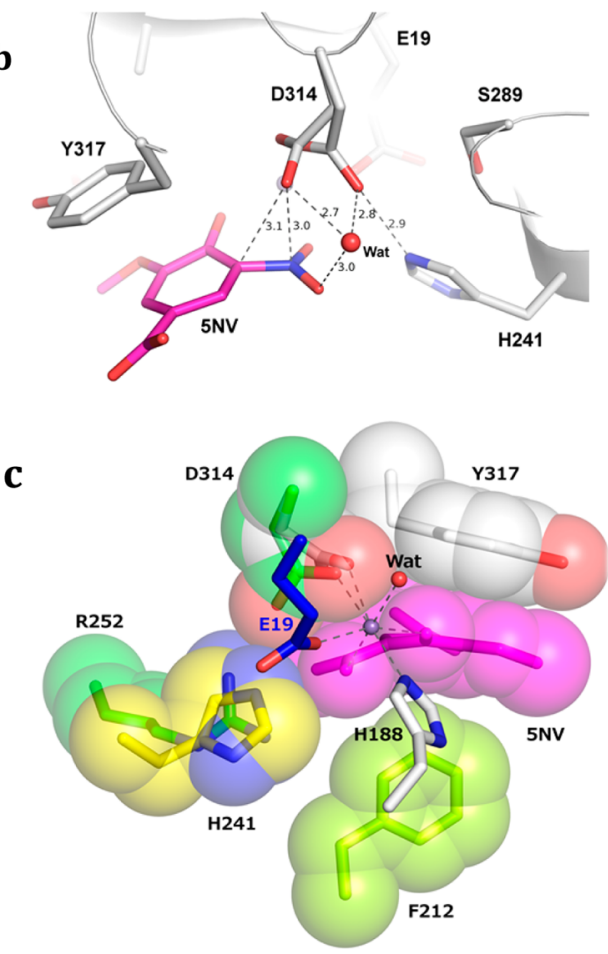

Figure 3. (a) $2 \mathrm{FoFc}$ map at a contour level of $\sigma=3.0$ is shown with 5$\mathrm{NV}$ (the carbon atoms are colored in magenta), selected amino acid side chains, and $\mathrm{Mn}^{2+}$ (the map contour level is $\sigma=5.0$ ) in the active site of LigW from N. aromaticivorans. (b) $\mathrm{Mn}^{2+}$ and 5-NV coordination in the active site. The distances are in Angstroms. (c) The "distorted" conformation of the inhibitor 5-NV (all atoms are colored magenta) represents a "perfect" fit in the LigW active site pocket (PDB id: $4 \mathrm{QRN}$ ). The amino acid side chains are shown as stick models and the semitransparent van der Waals spheres. Oxygen atoms are red, nitrogen atoms are blue, manganese is drawn as a gray nonbonded sphere, metal coordination bonds are shown as dotted gray lines. Hydrogen atoms were omitted for clarity.

\section{DISCUSSION}

The decarboxylation of 5-carboxyvanillate catalyzed by LigW requires the cleavage of the carbon-carboxylate bond at $\mathrm{C} 5$ of the substrate and protonation of the aromatic ring. Potential reaction mechanisms for this transformation are illustrated in Scheme 2. These mechanisms differ with respect to whether $\mathrm{C}-\mathrm{H}$ bond formation precedes or follows $\mathrm{C}-\mathrm{C}$ bond cleavage, and whether a water molecule is activated for nucleophilic attack with the substrate. Our results are most consistent with a reaction mechanism that requires the initial protonation of C5 of the substrate that is facilitated by a remarkable out-of-plane bending or distortion of the substrate by the enzyme.

Three-dimensional structures of LigW were determined from S. paucimobilis and $N$. aromaticivorans in the presence and absence of ligands bound in the active site. In general, the LigW structures obtained from these two sources are quite similar to
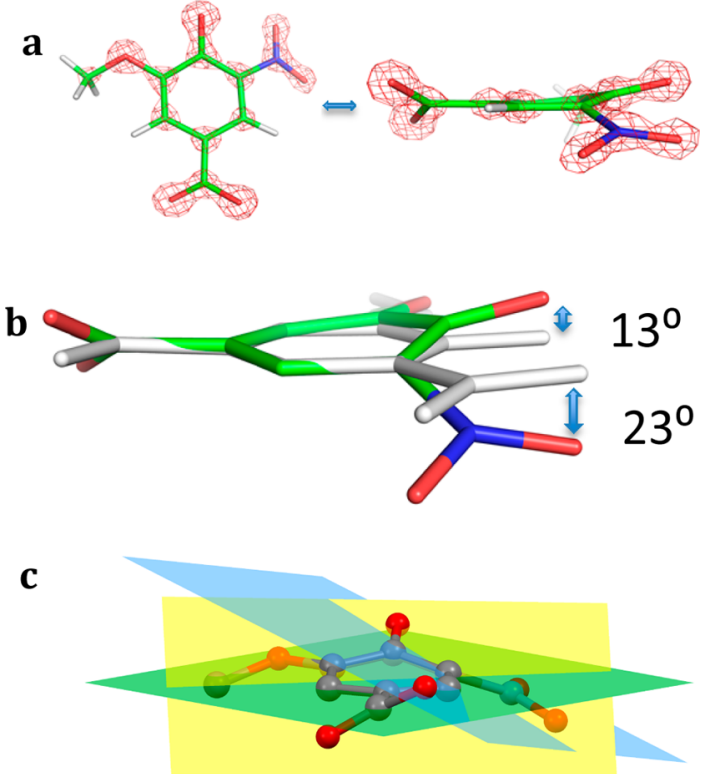

Figure 4. Geometric distortion of 5-NV in the active site of LigW from N. aromaticivorans (PDB id: 4QRN). (a) 5-NV structure shown as a stick model and the $2 \mathrm{FoFc}$ map (contour level $\sigma=3.0$, red colored) is drawn around it. Carbon atoms are green, oxygen red and nitrogen blue. (b) Superposition of coordinates for 5-NV bound to LigW (atoms are colored) and the small molecule 5-NV $0.96 \AA$ structure (all atoms are gray). (c) Distortion of enzyme-bound 5-NV, the planes are drawn through sets of atoms forming different planar groups: the ring plane (green; C1, C2, C6, carboxylate), the hydroxyl group plane (yellow; C3, C4, C5, methoxy group), and the nitro group plane (cyan; C5 and the nitro group).

one another but the structure of LigW complexed with 5-NV from $N$. aromaticivorans was determined to a resolution of 1.07 $\AA$ and thus this structure will serve as the foundation for the subsequent discussion about the reaction mechanism and activation of the substrate. The structure of LigW, with the tight-binding inhibitor 5-NV in the active site, diminishes the likelihood that a metal-activated water molecule is involved in the decarboxylation of 5-CV (PDB id: 4QRN). When 5-NV binds to the active site of LigW, two of the three water molecules that are initially bound to the manganese in the active site are displaced. The remaining water molecule is $4.4 \AA$ from $\mathrm{C} 4$ and $5.0 \AA$ from the nitro-group of the inhibitor and is ill-positioned to attack either the aromatic ring or the C5carboxylate group of the substrate. Therefore, mechanism a, as depicted in Scheme 2, is unlikely to be catalyzed by LigW. These considerations support a mechanism where protonation at C5 either precedes or follows the decarboxylation step (mechanisms $\mathbf{b}$ and $\mathbf{c}$ ).

All of the X-ray structures of LigW support the proposal that the hydrogen at $\mathrm{C} 5$ of the product vanillate is donated from either Asp-314 (Asp-296 in LigW from S. paucimobilis) or the hydroxyl group at $\mathrm{C} 4$ of the substrate. In the structure of LigW with 5-NV there are two alternate conformations for the carboxylate side chain of Asp-314. In these two conformations, one of the two carboxylate oxygens from the side chain of Asp314 is $3.1 \AA$ from C5 of the inhibitor. The closest alternative entity that could function as the proton donor is the hydroxyl substituent at C4. Asp-314 is conserved in all of the enzymes that have been functionally characterized in the amidohydrolase superfamily, and in those enzymes that catalyze hydrolytic 
reactions, this residue has been demonstrated to function as a general acid during protonation of the leaving group. ${ }^{5}$ Mutation of this residue to an asparagine results in the reduction of catalytic activity by more than 3 orders of magnitude and thus this residue is critical for the catalytic activity of LigW.

The most remarkable finding from the structure of LigW from $\mathrm{N}$. aromaticivorans, determined with $5-\mathrm{NV}$ in the active site, is the significant out-of-plane distortion of the substituent attached to C5 of the bound ligand. Similar distortions are also observed in the structures of LigW from S. paucimobilis determined with 5-NV, VAN, or MCB in the active site. The out-of-plane bending of the carboxylate substituent at C5 will facilitate the protonation of this carbon to the $s i$-face of the substrate from either the adjacent hydroxyl group at $\mathrm{C} 4$ or Asp314 . The very tight binding of the nitro-analogue (5-NV) of the substrate to $\operatorname{LigW}\left(K_{\mathrm{i}}=\sim 1.5-5.0 \mathrm{nM}\right)$ most likely reflects the resemblance of this compound to a reaction intermediate for the decarboxylation of 5-CV. We therefore propose that the distorted out-of-plane bending observed for the nitrosubstituent in 5-NV bound in the active site of LigW resembles the reaction intermediate (or the corresponding anion) depicted in Scheme 2c. This intermediate is formed by the delocalization of the electron pair from the ionized hydroxyl group at $\mathrm{C} 4$ to $\mathrm{C} 5$.

In reaction mechanisms $\mathbf{b}$ and $\mathbf{c}$ (Scheme 2), the proton that is ultimately found at $\mathrm{C} 5$ of the product vanillate is transferred either after the carboxylate group has been cleaved or before. For the reaction catalyzed by LigW it is apparent that protonation of $\mathrm{C5}$ prior to the decarboxylation step will require the delocalization of the electron pair from the neighboring phenolic oxygen at $\mathrm{C} 4$ (Scheme $2 \mathrm{c}$ ). In LigW, the critical participation of the hydroxyl group at $\mathrm{C} 4$ for the decarboxylation reaction is experimentally supported by the fact that we were unable to demonstrate that the substrate analogue that is missing the hydroxyl group at $\mathrm{C} 4$ (MCB) can be decarboxylated by LigW at a measurable rate. In the mechanism depicted in Scheme 2b, however, the hydroxyl group at C4 does not facilitate the decarboxylation reaction in any obvious manner. The X-ray structure of LigW from S. paucimobilis, determined in the presence of MCB (PDB id: 4NI8, Figure 2c), clearly demonstrates that the substrate analogue that is missing the hydroxyl group at $\mathrm{C} 4$ can bind to the active site of LigW in a manner that is nearly identical to that of the tight binding inhibitor 5-NV (Figure 2b) and the product vanillate (Figure 2d). Therefore, MCB is not a substrate because it is unable to bind to the active site of LigW.

We have also demonstrated that LigW can catalyze the exchange of the proton at $\mathrm{C} 5$ of the product vanillate with a rate constant of $7.9 \times 10^{-3} \mathrm{~s}^{-1}$ at $\mathrm{pD}$ 9.0. We were unable to observe any exchange with solvent over an incubation time of 7 days using 3-methoxybenzoate as a probe substrate with LigW. This observation further supports the proposal that the proton exchange reaction requires the participation of the hydroxyl group at $\mathrm{C} 4$ via the delocalization of the lone pair of electrons from the phenolate anion to C5.

The results from the product isotope effect (PIE) experiment conducted in an equal mixture of $\mathrm{H}_{2} \mathrm{O}$ and $\mathrm{D}_{2} \mathrm{O}$ also support the mechanism depicted in Scheme 2c. The PIE experiment provides a direct measurement of the partitioning of the anionic intermediate at $\mathrm{C} 5$ toward product formation. ${ }^{7}$ For the highly unstable anionic reaction intermediate depicted in Scheme $2 b$, irreversible protonation is the only possible outcome after the loss of $\mathrm{CO}_{2}$. Protonation of the anionic intermediate by Asp-
314 will be more rapid than proton exchange with bulk solvent. Therefore, the PIE for the mechanism depicted in Scheme $2 b$ is expected to be close to unity because there would effectively be no discrimination between the transfer of a proton and a deuteron to the highly unstable anionic intermediate. In contrast, the mechanism depicted in Scheme $2 \mathrm{c}$ can lead to a substantial PIE. In this mechanism, protonation of C5 occurs prior to the irreversible decarboxylation step. Therefore, the inherent kinetic discrimination between protonation and deuteration of the anionic intermediate at C5 in Scheme 2c will be fixed in the product vanillate after the subsequent irreversible decarboxylation step. The PIE of 4.6 for LigW therefore supports a mechanism where protonation at $\mathrm{C5}$ occurs prior to the decarboxylation step.

The proposed mechanism of action for the decarboxylation of 5-carboxyvanillate by LigW is presented in Scheme 3. In this

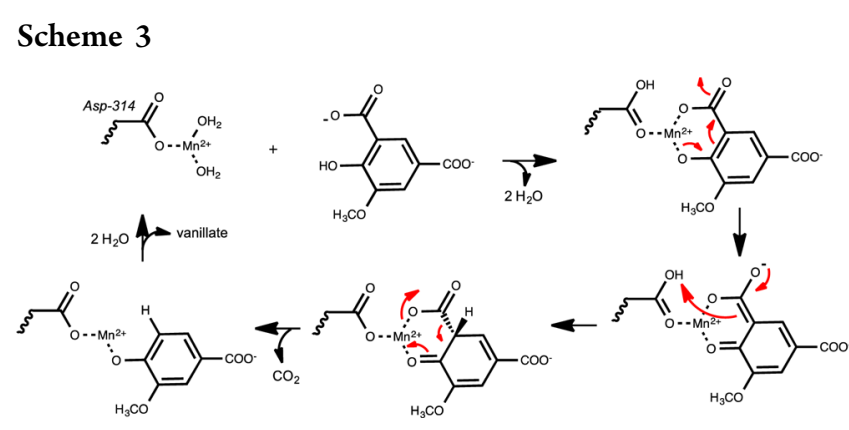

mechanism the binding of substrate to the active site of LigW results in the displacement of two water molecules from the mononuclear metal center in the active site of the enzyme. The hydroxyl group at C4 and the carboxylate group at C5 are directly coordinated to the divalent cation. The formation of this complex is fully supported by the X-ray structures of complexes of LigW crystallized in the presence of 5-NV, VAN, and MCB. The binding of the substrate in the active site is accompanied by an out-of-plane bending of the substituent at $\mathrm{C} 5$ of the substrate and a reduction in the $\mathrm{p} K_{\mathrm{a}}$ for the ionization of the hydroxyl group at C4. The proton from the hydroxyl group is subsequently transferred to the side chain carboxylate from Asp-314 or directly to C5 after delocalization of the lone pair of electrons from the ionized hydroxyl group to C5. Decarboxylation follows the protonation of $\mathrm{C5}$ but it is uncertain whether or not the phenolate substituent is protonated before, or after, the product leaves the active site. Similar reaction mechanisms can be proposed for the other known decarboxylases from $\operatorname{cog} 2159$, including $\gamma$-resorcylate decarboxylase, ${ }^{10}$ isoorotate decarboxylase, ${ }^{11}$ and aminocarboxymuconate semialdehyde decarboxylase. ${ }^{12}$

The reaction catalyzed by orotidine 5 -monophosphate decarboxylase (OMPDC) is superficially similar to that of LigW. However, OMPDC does not utilize a metal ion in the active site and the structure of orotidine precludes the protonation of the substrate prior to the decarboxylation step. In the decarboxylation reaction catalyzed by OMPDC, the product isotope effect was determined to be 1.0 and the reaction mechanism has been proposed to occur via formation of an unstable vinyl carbanion intermediate where carboncarbon bond cleavage precedes carbon-hydrogen bond formation. ${ }^{7}$ OMPDC can also catalyze the exchange of the proton at $\mathrm{C} 6$ of the product UMP. ${ }^{8}$ Recent high-resolution structures of OMPDC determined in the presence of substrate 
analogues have shown that this enzyme may facilitate the decarboxylation of orotidine 5 -phosphate by distortion of the carboxylate group attached to C6 of the substrate. ${ }^{9}$

In this paper we have not addressed whether the initial reaction product of the $\mathrm{LigW}$ catalyzed reaction is either $\mathrm{CO}_{2}$ or $\mathrm{HCO}_{3}{ }^{-}$. Kluger has argued for the preferential cleavage of bicarbonate over carbon dioxide, in some cases, based upon the high reactivity of $\mathrm{CO}_{2}$ and the probability of internal return back to substrate, relative to product dissociation. ${ }^{13}$ In the structure of LigW from $N$. aromaticivorans there is a single water molecule that is positioned to potentially attack the $\mathrm{C5}$ carboxylate of the substrate (Figure $3 \mathrm{~b}$ ). This water molecule is $3.8 \AA$ from the carboxylate carbon and positioned at an angle of $\sim 44^{\circ}$ above the plane of the carboxylate. It is also in hydrogen bonding distance to the side chain of Asp-314. Recently, Xu et al. have proposed that the reaction mechanism catalyzed by isoorotate decarboxylase (a structurally related decarboxylase from the amidohydrolase superfamily) is initiated by either the attack of a water molecule on the carboxylate group that is to be cleaved or by the side chain of the aspartate that is structurally equivalent to Asp-314 in LigW. ${ }^{11}$ However, the mechanisms proposed by $\mathrm{Xu}$ et al. are deficient since they do not address how C5 of the product uracil is ultimately protonated. ${ }^{11}$ With LigW, experiments are in progress to directly measure the rate of formation of $\mathrm{CO}_{2}$ by membrane inlet mass spectrometry. ${ }^{14}$

Most of the residues contained within the active site of LigW are critical for catalytic activity of the enzyme. Mutation of either of the two arginine residues that ion-pair with the carboxylates at $\mathrm{C} 1$ and $\mathrm{C} 5$ diminishes the value of $k_{\text {cat }} / K_{\mathrm{m}}$ by approximately 3 orders of magnitude. Mutation of the tyrosine that is $\pi$-stacked against the aromatic ring of the substrate results in the loss of activity of nearly 4 orders of magnitude. Mutation of the putative proton transfer residue, Asp-314 (Asp296 in LigW from S. paucimobilis), diminishes the catalytic activity by more than 3 orders of magnitude. The X-ray structure of LigW has revealed a hydrogen-bonded network from Asp-314 to His-242 and Glu-245 (His-226 and Glu-229 in the $S$. paucimobilis LigW). These two residues are also conserved in other decarboxylases from $\operatorname{cog} 2159$ and mutation of these residues diminishes the value of $k_{\text {cat }} / K_{\mathrm{m}}$ significantly. The specific catalytic function of this hydrogen-bonded network is unclear, but these residues may be involved in the activation of the water molecule observed in Figure $3 \mathrm{~b}$. These residues may modulate the $\mathrm{pK}_{\mathrm{a}}$ of Asp-314, function as a conduit for protons to and from the active site, or aid in maintaining the active site architecture. The $\mathrm{pH}$-rate profiles of LigW are bell-shaped where one residue must be ionized for optimal catalytic activity and another residue protonated. The loss of activity at low $\mathrm{pH}$ likely reflects the protonation of Asp314 and the ionization at high $\mathrm{pH}$ likely reflects the ionization of His-241, since this is the only other residue within the active site that can be protonated in this range of $\mathrm{pH}$ values.

Since the introduction of transition-state theory to enzymecatalyzed reactions, it has generally been acknowledged that intermediates and transition states bind more strongly to the enzyme than does the substrate. ${ }^{15}$ During catalysis, the Michaelis complex can undergo a series of conformational reorganizations that facilitate the transformation from substrate to product. These conformational transitions are challenging to observe due to the very short life times of the intermediates involved and the high resolution that is required to detect the frequently small changes in bond distances and dihedral angles. $^{16,17}$ However, lysozyme ${ }^{18}$ and other glycoside hydro- lases ${ }^{19}$ have been shown to catalyze the hydrolysis of polysaccharides by distorting the sugar moiety into a strained half-chair conformation where the glycosidic bond becomes more susceptible to hydrolysis. Enzyme-substrate complexes with distorted substrate geometries have also been reported for other enzymes including transketolase, ${ }^{20,21}$ translaldolase, ${ }^{22}$ and tyrosine phenol-lyase, ${ }^{23}$ among others. In many cases, these strained conformations can only be obtained by site-specific mutations that reduce the catalytic rate and allow for trapping of relatively unstable intermediates. In this paper we have demonstrated that the reaction catalyzed by LigW is enhanced by the substantial distortion of the substrate prior to the decarboxylation of the substrate.

\section{MATERIALS AND METHODS}

Materials. 5-nitrovanillate (5-NV) was synthesized according to published procedures. ${ }^{24}$ Pfu Turbo DNA polymerase and the Escherichia coli strains BL21(DE3) and XL1-blue cells were obtained from Stratagene. The restriction enzyme DpnI was purchased from New England BioLabs and oligonucleotides were obtained from Integrated Data Technology through the Gene Technology Laboratory at Texas $\mathrm{A} \& \mathrm{M}$ University. Kanamycin, isopropyl $\beta$-Dthiogalactopyranoside (IPTG), and LB broth were acquired from Research Products International Corp. Protamine sulfate, Wizard Plus SV Miniprep DNA purification system, and Vivaspin centrifugal concentrator (10000 MWCO) were obtained from MP Biomedicals LLC., Promega, and Fisher Scientific, respectively.

Expression and Purification of LigW from S. paucimolbilis. The recombinant plasmid SpCD00297569 (NYSGXRC clone ID: 9453b1BCt3p1) bearing the gene for LigW (gil19110430) from $S$. paucimobilis was obtained through the PSI Material Repository (dnasu. asu.org). The NYSGXRC clone encoded codon-optimized LigW with an N-terminal Met-Ser-Leu insertion and a C-terminal Glu-Gly-His 6 . The nucleotides coding for the His-tag were removed from the plasmid using standard site directed mutagenesis techniques with the complementary primer pair 5'- CGCATCACCTAATGACATCATCACCATCACCACTG-3' and 5'-CAGTGGTGATGGTGATGATGTCATTAGGTGATGCG- $3^{\prime}$. The QuikChange reaction product was transformed into E. coli XL1-blue cells and the plasmid was isolated using the Wizard Plus SV Miniprep DNA purification system. After verifying the DNA sequence, the expression plasmid was transformed into E. coli BL21(DE3) cells by electroporation and then grown on LB-agar plates containing $50 \mu \mathrm{g} / \mathrm{mL}$ kanamycin for $14 \mathrm{~h}$ at $37{ }^{\circ} \mathrm{C}$. A single colony was grown overnight in $7 \mathrm{~mL}$ of $\mathrm{LB}$ medium containing $50 \mu \mathrm{g} / \mathrm{mL}$ kanamycin at $37^{\circ} \mathrm{C}$. The cell culture was used to inoculate $1 \mathrm{~L}$ of the same medium, which was further allowed to incubate by agitation at $37^{\circ} \mathrm{C}$. The temperature was lowered to $25^{\circ} \mathrm{C}$ after the optical density at $600 \mathrm{~nm}$ reached 0.6 , at which point protein expression was induced with $0.5 \mathrm{mM}$ IPTG in the presence of $1.0 \mathrm{mM}$ $\mathrm{MnCl}_{2}$. The cells were harvested by centrifugation $\left(12000 \mathrm{~g}\right.$ at $4{ }^{\circ} \mathrm{C}$ for $10 \mathrm{~min}$ ) after $14 \mathrm{~h}$ of incubation. The cell pellet was resuspended in 20 $\mathrm{mM}$ HEPES at $\mathrm{pH} 7.3$ and then lysed by sonication using $5 \mathrm{~s}$ pulses for $20 \mathrm{~min}$ at $0{ }^{\circ} \mathrm{C}$ in the presence of $0.2 \mathrm{mg} / \mathrm{mL}$ phenylmethanesulfonyl fluoride (PMSF). The cellular debris was separated by centrifugation and the supernatant fluid treated with $10 \mathrm{~mL} 2 \%$ (w/ v) protamine sulfate for $30 \mathrm{~min}$ to precipitate the nucleic acids. The protein was precipitated with $45 \%$ saturation of solid ammonium sulfate, resuspended in a minimal amount of $20 \mathrm{mM}$ HEPES ( $\mathrm{pH} 7.3$ ), filtered with a $0.2 \mu \mathrm{m}$ cellulose acetate syringe filter, and loaded onto a Hiload 26/60 Superdex 200 prep grade gel filtration column (Amersham Biosciences). The purity of the protein was confirmed by SDS-PAGE. The fractions were pooled and concentrated to $17 \mathrm{mg} /$ $\mathrm{mL}$ using a Vivaspin centrifugal concentrator (10000 MWCO) at $4500 \mathrm{rpm}$.

Crystallization of LigW from S. paucimobilis. Crystals of LigW from S. paucimobilis liganded with $\mathrm{Mn}^{2+}$ were grown using the sitting drop method at room temperature. The protein solution contained enzyme $(17 \mathrm{mg} / \mathrm{mL})$ in $20 \mathrm{mM}$ HEPES ( $\mathrm{pH} 7.3$ ), and $1.0 \mathrm{mM}$ 
$\mathrm{MnCl}_{2}$; the precipitant contained 20\% PEG 10000, 0.10 M HEPES ( $\mathrm{pH} 7.5$ ), and $1.0 \mathrm{mM} \mathrm{MnCl}_{2}$. Crystals appeared in 3 days and exhibited diffraction consistent with the space group P1, with eight subunits per asymmetric unit. The crystals of LigW liganded with $\mathrm{Mn}^{2+}$ and vanillic acid were grown by the sitting drop method at room temperature. The protein solution contained enzyme $(17 \mathrm{mg} / \mathrm{mL})$ in $20 \mathrm{mM}$ HEPES ( $\mathrm{pH} 7.3$ ), $1.0 \mathrm{mM} \mathrm{MnCl}{ }_{2}$, and $160 \mathrm{mM}$ vanillic acid. The precipitant contained 20\% PEG 10000, 0.10 M HEPES ( $\mathrm{pH} 7.5$ ), and $1.0 \mathrm{mM} \mathrm{MnCl}$. Crystals appeared in 1 week and exhibited diffraction consistent with the space group P1, with eight polypeptides per asymmetric unit. The crystals of LigW liganded with $\mathrm{Mn}^{2+}$ and 5nitrovanillate were grown by the sitting drop method at room temperature. The protein solution contained enzyme $(17 \mathrm{mg} / \mathrm{mL})$ in $20 \mathrm{mM}$ HEPES ( $\mathrm{pH} 7.3$ ), $1.0 \mathrm{mM} \mathrm{MnCl}_{2}$, and $2.0 \mathrm{mM} \mathrm{HMN}$. The precipitant contained 20\% PEG 10000, 0.1 M HEPES (pH 7.5), and $1.0 \mathrm{mM} \mathrm{MnCl}$. Crystals appeared in 3 days and exhibited diffraction consistent with the space group P1, with eight polypeptides per asymmetric unit. The crystals of LigW liganded with $\mathrm{Mn}^{2+}$ and 3methoxy-5-carboxybenzoate were grown by the sitting drop method at room temperature. The protein solution contained enzyme $(17 \mathrm{mg} /$ $\mathrm{mL}$ ) in $20 \mathrm{mM}$ HEPES (pH 7.3), $1.0 \mathrm{mM} \mathrm{MnCl}_{2}$, and $80 \mathrm{mM} \mathrm{MCB}$. The precipitant contained 20\% PEG 10000, 0.10 M HEPES ( $\mathrm{pH} 7.5$ ), and $1.0 \mathrm{mM} \mathrm{MnCl}$. Crystals appeared in 10 days and exhibited diffraction consistent with the space group $\mathrm{P} 1$, with eight polypeptides per asymmetric unit. Prior to data collection, the crystals of the four LigW complexes were transferred to cryoprotectant solutions composed of their mother liquids and $20 \%$ glycerol and flash-cooled in a nitrogen stream. Four X-ray diffraction data sets (Table S2) were collected at the NSLS X29A beamline (Brookhaven National Laboratory) on the $315 \mathrm{Q}$ CCD detector. Diffraction intensities were integrated and scaled with programs DENZO and SCALEPACK. ${ }^{25}$ The data collection statistics are given in Table S2.

Structure Determination and Model Refinement of LigW from S. paucimobilis. Four LigW structures were solved by molecular replacement with fully automated molecular replacement pipeline BALBES, ${ }^{26}$ using only input diffraction and sequence data. BALBES used the structure of $\gamma$-resorcylate decarboxylase $(\gamma$-RSD) with the PDB id: 2DVT as the search model. Partially refined structures of the four LigW crystal forms were the outputs from BALBES. Subsequent iterative cycles of refinement were performed for each crystal form including: model rebuilding with COOT, ${ }^{27}$ refinement with PHENIX, ${ }^{28}$ and automatic model rebuilding with ARP. ${ }^{29}$ The quality of the final structures was verified with omit maps. The stereochemistry was checked with WHATCHECK ${ }^{30}$ and MOLPOBITY. ${ }^{31}$ The program LSQKAB ${ }^{32}$ was used for structural superposition. Structure figures with electron density maps were prepared using PYMOL. ${ }^{33}$ The final models of the unliganded and liganded crystal forms of LigW contain all 334 residues of the enzyme in all eight subunits in the asymmetric unit. The $\mathrm{Mn}^{2+}$ ion is welldefined in the active site of every monomer. Final crystallographic refinement statistics for all determined LigW structures are provided in the Table S2.

Cloning, Expression, and Purification of LigW from $N$. aromaticivorans. A pNIC28-Bsa4 vector containing the gene for LigW (UNP ID: Q2GA79, locus tag: Saro_0799) was transformed into BL21(DE3) E. coli containing the pRIL plasmid (Stratagene) and then used to inoculate an overnight culture containing $25 \mu \mathrm{g} / \mathrm{mL}$ kanamycin and $34 \mu \mathrm{g} / \mathrm{mL}$ chloramphenicol. The culture was allowed to grow overnight at $37^{\circ} \mathrm{C}$ in a shaking incubator. One $\mathrm{mL}$ of the overnight culture was used to inoculate $1 \mathrm{~L}$ of PASM-5052 autoinduction medium containing $100 \mu \mathrm{g} / \mathrm{mL}$ kanamycin and 34 $\mu \mathrm{g} / \mathrm{mL}$ chloramphenicol. The culture was placed in a LEX48 airlift fermenter and incubated at $37^{\circ} \mathrm{C}$ for $4 \mathrm{~h}$ and then at $22{ }^{\circ} \mathrm{C}$ overnight. The culture was harvested and pelleted by centrifugation and stored at $-80{ }^{\circ} \mathrm{C}$ until needed.

The cells were resuspended in lysis buffer (20 mM HEPES, $\mathrm{pH} 7.5$, $500 \mathrm{mM} \mathrm{NaCl}, 20 \mathrm{mM}$ imidazole, and $10 \%$ glycerol) and lysed by sonication. The lysate was clarified by centrifugation at $35,000 \mathrm{~g}$ for 30 min. The clarified lysate was loaded onto a $1 \mathrm{~mL}$ HisTrap FF column, washed with 10 column volumes of lysis buffer, and eluted in buffer containing $20 \mathrm{mM}$ HEPES, pH 7.5, $500 \mathrm{mM} \mathrm{NaCl}, 500 \mathrm{mM}$ imidazole, and $10 \%$ glycerol. The purified sample was loaded onto a HiLoad S200 16/60 PR gel filtration column, which was equilibrated with SECB buffer (20 mM HEPES, pH 7.5, $150 \mathrm{mM} \mathrm{NaCl}, 10 \%$ glycerol, and $5 \mathrm{mM}$ DTT). Peak fractions were collected, concentrated by centrifugation, analyzed by SDS-PAGE, snap frozen in liquid nitrogen, and stored at $-80{ }^{\circ} \mathrm{C}$.

Crystallization and Structure Determination of LigW from $N$. aromaticivorans. Wild-type LigW was crystallized by the sitting-drop vapor diffusion method. The protein $(0.4-1.0 \mu \mathrm{L}$ of a $12-30 \mathrm{mg} / \mathrm{mL}$ solution) was mixed with an equal volume of a precipitant solution and equilibrated at $21{ }^{\circ} \mathrm{C}$ against the same precipitant solution in clear tape-sealed 96-well INTELLI-plates (Art Robbins Instruments, Sunnyvale, CA). Crystallization was performed using a PHOENIX crystallization robot (Art Robbins Instruments) and four types of MCSG commercial crystallization screens (Microlytic North America, Burlington MA, USA). The appearance of protein crystals was monitored either by manual inspection or using a RockImager 1000 (Formulatrix, Waltham, MA) starting within $24 \mathrm{~h}$ of incubation. The protein crystals were obtained using two sets of precipitants. The first set was $0.17 \mathrm{M}$ ammonium acetate, $0.085 \mathrm{M}$ sodium citrate- $\mathrm{HCl}$ ( $\mathrm{pH}$ 5.6), 25.5\% (w/v) PEG 4000, and $15 \%(\mathrm{v} / \mathrm{v})$ glycerol, and the second set was $0.085 \mathrm{M}$ Tris- $\mathrm{HCl}, \mathrm{pH} 8.5,0.17 \mathrm{M}$ sodium acetate, $25 \%(\mathrm{w} / \mathrm{v})$ PEG 4000, and $15 \%(\mathrm{v} / \mathrm{v})$ glycerol. The crystal structures produced with both sets of precipitants were nearly the same and the final choice of the structures was based on quality and resolution of X-ray diffraction data. To produce crystals of protein-ligand complexes, we have used the cocrystallization method (the ligands were added to protein solutions before crystallization, for instance, $1.0 \mathrm{mM} \mathrm{MnCl}$

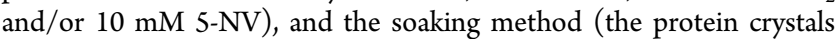
were soaked in the precipitant-based ligand solutions for 1-24 h) to produce the structures of LigW complexes with manganese and 5-NV. There were no significant differences between the structures produced by both methods. However, the crystal quality (based on higher resolution and lower mosaicity of the X-ray diffraction data) was better when the cocrystallization method was used. All the structures reported here were the results of cocrystallization experiments. The crystals of the wild-type LigW-Mn complex were obtained using the precipitant set-1, and the crystals of the wild-type LigW-Mn-5-NV complex were grown using the precipitant set-2. The crystals of the D314N LigW-Mn-5-NV mutant complex were obtained using the precipitant set-1 supplemented with $1 \mathrm{mM} \mathrm{MnCl}_{2}$ and $10 \mathrm{mM} 5-\mathrm{NV}$, and the crystals of the D314N mutant in complex with 5-NV only were obtained using the precipitant set-1, supplemented with $10 \mathrm{mM}$ 5-NV only. The rod- or plate-shaped protein crystals grew in 24-48 h and the crystals with 5-NV were light-brown colored. All crystals were directly flash-frozen and stored in liquid nitrogen until use.

$\mathrm{X}$-ray diffraction data were collected from the frozen crystals at $93 \mathrm{~K}$ on the beamline X29A (National Synchrotron Light Source (NSLS), Brookhaven National Laboratory, Upton, NY) at a wavelength of 0.92 or $1.075 \AA$. All data were processed and scaled with HKL2000. ${ }^{26}$ The first crystal structure of LigW was determined by molecular replacement using coordinates of $\gamma$-resorcylate decarboxylase (PDB id: 2DVT) as a search model and a PHASER software embedded in the CCP4 program package suite. ${ }^{32}$ All subsequent structures were solved using coordinates of a solved LigW structure as a search model. The initial model was built and refined by ARP/WARP. ${ }^{29}$ Each structure, except one, was further refined using the program REFMAC $^{34}$ using the diffraction data as structure factor amplitudes.

The $1.07 \AA$ structure of the LigW-Mn-5NV complex was refined using a PHENIX automated structure solution and refinement platform ${ }^{28}$ and the diffraction data as structure factor intensities, not amplitudes. The initial set of geometric restraints for refinement of 5$\mathrm{NV}$ was created on the basis of the $0.96 \AA 5$-NV crystal structure (Table S4) including corrections for $\mathrm{C}-\mathrm{O}$ distances in carboxyl groups based on $\mathrm{pH}$ difference in crystallization conditions. In the last refinement cycle the coordinates of 5-NV were refined without any geometric restraints. Noncrystallographic symmetry restraints (NCS) were not used in refinement. All atom occupancies, where applicable, were refined in groups, the occupancies for $\mathrm{Mn}^{2+}$ ions were refined 
individually. All hydrogen atoms were refined as riding hydrogens. We have found it useful to disallow zero occupancy values assigned by PHENIX for (hydroxyl group) hydrogen atoms of serine and threonine-all hydrogen were given occupancies of either 1.0 (or 0.99 ) and refined in groups with all other atoms of the same amino acid residue. All the models were verified and fixed manually using COOT visualization and refinement program. ${ }^{27}$ Metal coordination bonds were refined without geometric restraints.

The data collection and final refinement statistics of the protein crystal structures are presented in Table S3. The coordinates and structure factors were deposited to the Protein Database: PDB id: 4QRN for the Mn-5-NV complex; PDB id: 4QTG for the Mncomplex, PDB id: 4QS5 for the D314N mutant, Mn-5-NV complex; and PDB id: 4QS6 for the D314N mutant, Mn-complex. The figures were generated by PYMOL. ${ }^{33}$

Structure Determination of 5-CV and 5-NV. The substrate 5$\mathrm{CV}$ and the inhibitor 5-NV alone were crystallized and their threedimensional structures determined. Each compound (2-4 mg) was dissolved in $50 \mu \mathrm{L}$ of water supplemented with $5 \%(\mathrm{v} / \mathrm{v})$ glycerol and then warmed to $80{ }^{\circ} \mathrm{C}$ for 3-4 min. The solutions were cleared from insoluble material by centrifugation ( $1 \mathrm{~min}$ at $16,000 \mathrm{rpm}$ ). Sittingdrop crystallization was performed using 24-well Linbro plates (Hampton Research). Each ligand solution $(2-5 \mu \mathrm{L})$ was equilibrated against a precipitant, consisting of $80 \%(\mathrm{v} / \mathrm{v})$ glycerol and $20 \%$ water for a few hours. The thin $(\sim 20-30 \mu \mathrm{m})$ and very long (up to a few $\mathrm{mm}$ ) colorless crystals of 5-CV grew over a period of 10-120 min depending on the crystallization drop size. The faster appearing crystals (in smaller drops) tended to stick together and grow as large clusters, which were difficult to separate. The crystals in larger drops, however, grew slower and were less clustered. Single crystals were flash frozen and stored in liquid nitrogen until data collection. 5-NV was crystallized in a similar fashion; however, the very small plate-like crystals that appeared after 10-40 min of incubation diffracted poorly. The large $(0.2 \times 0.2 \times 0.3 \mathrm{~mm})$ yellow crystals grew in a few hours. These crystals were also frozen and kept in liquid nitrogen until use.

The X-ray diffraction data from the frozen crystals were collected at $93 \mathrm{~K}$ on the beamline X29A (National Synchrotron Light Source, Brookhaven National Laboratory, Upton, NY) at a wavelength of 0.92 Å using a CBASS software. A total of 72 frames were collected for each data set using a $5^{\circ}$ oscillation range (a total of $360^{\circ}$ rotation). The crystals of 5-CV and 5-NV diffracted to a resolution of 0.96 and 0.90 $\AA$, respectively. The data were processed and scaled using XDS. ${ }^{35}$ The structure factor files were converted into a SHELX format using the program XPREP. ${ }^{36}$ The structures were solved by SHELXS and then refined using SHELXL ${ }^{37}$ with the help of the WinGX program package and visualization software. ${ }^{38}$ Geometry was analyzed using the program MERCURY 3.3. ${ }^{39}$ All figures for small molecules were generated by MERCURY. The crystal and data collection, and refinement statistics and geometric parameters for 5-CV and 5-NV are listed in Tables S4 and S5, respectively.

Molecular Weight Determinations. The oligomeric state of LigW was determined using a 10/300 GL Superdex 200 column from GE Healthcare calibrated with gel filtration standards (Bio-Rad Laboratories, Inc.) containing a lyophilized mixture of thyroglobulin (MW 670000 ), bovine $\gamma$-globulin (MW 158000 ), chicken ovalbumin (MW 44000), equine myoglobin (MW 17000), and vitamin B12 (MW 1,350) in $50 \mathrm{mM}$ HEPES, $\mathrm{pH}$ 7.0. Various concentrations of LigW ranging from $0.5 \mathrm{mg} / \mathrm{mL}$ to $2.0 \mathrm{mg} / \mathrm{mL}$ were utilized at a flow rate of $0.5 \mathrm{~mL} / \mathrm{min}$ with a pressure of $1.5 \mathrm{MPa}$.

Metal Analysis. The metal content of LigW was determined by inductively coupled plasma mass spectrometry (ICP-MS) with an Elan DRC II ICP-MS instrument from PerkinElmer. Prior to ICP-MS analysis, loosely bound metals were removed by passage through a PD10 desalting column equilibrated with $50 \mathrm{mM}$ HEPES ( $\mathrm{pH} 7.5$ ) previously treated with Chelex 100 ion-exchange resin. The protein sample was treated with concentrated $\mathrm{HNO}_{3}$ for $15 \mathrm{~min}$ at $100{ }^{\circ} \mathrm{C}$ and then diluted with distilled water to a final protein concentration of 1.0 $\mu \mathrm{M}$ and $1 \%(\mathrm{v} / \mathrm{v}) \mathrm{HNO}_{3}$. The activation of $\mathrm{LigW}$ by $\mathrm{Mn}^{2+}, \mathrm{Zn}^{2+}, \mathrm{Co}^{2+}$, or $\mathrm{Fe}^{2+}$ was analyzed by the addition of these metal ions directly to buffered solutions of the enzyme at $\mathrm{pH} 7.3$ and the catalytic activity was monitored after $12 \mathrm{~h}$ of incubation at $4{ }^{\circ} \mathrm{C}$. Iron was incubated anaerobically under argon prior to measurement of catalytic activity. Metal-free LigW was prepared by dialysis against $10 \mathrm{mM} O$ phenanthroline at $4{ }^{\circ} \mathrm{C}$ in $50 \mathrm{mM}$ HEPES, pH 6.5. A PD-10 column, previously equilibrated with $50 \mathrm{mM}$ HEPES at $\mathrm{pH} 7.3$, was used to remove the $o$-phenanthroline prior to analysis by ICP-MS.

Site-Directed Mutagenesis. All single site mutations to LigW were constructed following the QuikChange site-directed mutagenesis instruction manual of the manufacturer. The reaction product was transformed into E. coli XL1-blue cells and the plasmid isolated using the Wizard Plus SV Miniprep DNA purification system. After verifying the DNA sequence, all mutants underwent standard protein expression and purification as described previously for wild-type LigW.

Kinetic Parameters. The decarboxylation of 5-carboxyvanillate was determined by monitoring the decrease in absorbance upon formation of vanillate at $312 \mathrm{~nm}$ using a differential molar extinction coefficient $\left(\Delta \varepsilon_{312}\right)$ of $2,040 \mathrm{M}^{-1} \mathrm{~cm}^{-1}$. All assays were conducted using a SpectraMax Plus ${ }^{384}$ UV-vis spectrophotometer and the steadystate kinetic parameters were obtained using SoftMax Pro 5.0. Standard assay conditions for the decarboxylation of 5-CV included $50 \mathrm{mM}$ HEPES (pH 7.3), $1.0 \mathrm{mM} \mathrm{MnCl}$, and $0.1 \mu \mathrm{M} \mathrm{LigW} \mathrm{in} \mathrm{a} \mathrm{final}$ volume of $250 \mu \mathrm{L}$ at $30^{\circ} \mathrm{C}$. The kinetic constants were obtained using eq 1 , where $v$ is the initial velocity, $E_{t}$ is the total enzyme concentration, $[\mathrm{A}]$ is the substrate concentration, $k_{\mathrm{cat}}$ is the turnover number, and $K_{\mathrm{m}}$ is the Michaelis constant.

$$
v / E_{t}=k_{\text {cat }}[\mathrm{A}] /\left(K_{\mathrm{m}}+[\mathrm{A}]\right)
$$

Measurement of pH-Rate Profiles. The kinetic constants for wild-type $\mathrm{LigW}$ were determined as a function of $\mathrm{pH}$ from 5.0 to 9.0 The $\mathrm{pH}$ dependence of $k_{\text {cat }}$ and $k_{\text {cat }} / K_{\mathrm{m}}$ was determined in $50 \mathrm{mM}$ MES (pH 5.5-6.75), HEPES ( $\mathrm{pH} 7.0-8.25$ ), and CHES ( $\mathrm{pH}$ 8.59.0 ) buffers. The assays were conducted at approximately $0.25 \mathrm{pH}$ unit intervals and the final $\mathrm{pH}$ was recorded after completion of the reaction. Differential molar extinction coefficients for the conversion of substrate to product were determined at each $\mathrm{pH}$ value; these ranged from $\left(\Delta \varepsilon_{312}=2,109 \mathrm{M}^{-1} \mathrm{~cm}^{-1}\right)$ at $\mathrm{pH} 5.5$ to $\left(\Delta \varepsilon_{312}=205 \mathrm{M}^{-1} \mathrm{~cm}^{-1}\right)$ at $\mathrm{pH}$ 9.0. The kinetic data were fit to eq 2 using SigmaPlot 11.0, where $y$ is either $k_{\mathrm{cat}}$ or $k_{\mathrm{cat}} / K_{\mathrm{m}}, c$ is the $\mathrm{pH}$-independent value of $y$, $[\mathrm{H}]$ is the proton concentration, and $K_{\mathrm{a}}$ and $K_{\mathrm{b}}$ are the acid dissociation constants for the ionizing group at low and high $\mathrm{pH}$, respectively.

$$
\log y=\log \left(c /\left(1+\left([\mathrm{H}] / K_{\mathrm{a}}\right)+\left(K_{\mathrm{b}} /[\mathrm{H}]\right)\right)\right)
$$

Inhibition Studies. Vanillate, 3-methoxy-5-carboxybenzoate, and 5-nitrovanillate were utilized as inhibitors for the reaction catalyzed by LigW. The data for the tight-binding inhibition the enzyme by 5-NV were fit to eq 3 , where $E_{t}$ is the total enzyme concentration, $[\mathrm{I}]$ is the inhibitor concentration, $v_{0}$ is initial enzyme activity in the absence of inhibitor, $v_{\mathrm{i}}$ is the activity of the enzyme in the presence of inhibitor and $K_{\mathrm{i}}^{\text {app }}$ is the apparent inhibition constant. ${ }^{40,41}$ The dissociation constant, $K_{\mathrm{d}}$, for the binding of 5-CV to LigW was obtained using eq 4, where $A$ is the substrate concentration used in the inhibition experiment and $K_{\mathrm{m}}$ is the Michaelis constant for the substrate 5carboxyvanillate. $^{40,41}$ The inhibition experiments for 5-NV contained $600 \mu \mathrm{M}$ 5-carboxyvanillate, $50 \mathrm{mM}$ HEPES (pH 7.3), $1.0 \mathrm{mM} \mathrm{MnCl}_{2}$ and $0.1 \mu \mathrm{M} \mathrm{LigW} \mathrm{in} \mathrm{a} \mathrm{total} \mathrm{volume} \mathrm{of} 250 \mu \mathrm{L}$ at $30^{\circ} \mathrm{C}$. The apparent inhibition constants $\left(K_{\mathrm{i}}^{\text {app }}\right)$ for vanillate and 3-methoxy-5-carboxybenzoate were obtained from a fit of the data to eq 5 and the $K_{\mathrm{d}}$ was obtained from eq 4 . These experiments were conducted at a concentration of 5-carboxyvanillate of $80 \mu \mathrm{M}$.

$$
\begin{aligned}
v_{i} / v_{0}= & \left(\left[E_{t}\right]-K_{\mathrm{i}}^{\mathrm{app}}-[\mathrm{I}]\right. \\
& \left.+\left(\left([\mathrm{I}]+K_{\mathrm{i}}^{\mathrm{app}}-\left[E_{t}\right]\right)^{2}+\left(4 K_{\mathrm{i}}^{\mathrm{app}}\left[E_{t}\right]\right)\right)^{1 / 2}\right) /\left(2\left[E_{t}\right]\right) \\
K_{\mathrm{i}}^{\mathrm{app}}= & K_{\mathrm{d}}\left(1+[\mathrm{A}] / K_{\mathrm{m}}\right) \\
v_{i} / v_{0}= & K_{\mathrm{i}}^{\mathrm{app}} /\left(I+K_{\mathrm{i}}^{\mathrm{app}}\right)
\end{aligned}
$$


Solvent Isotope Effects. Steady-state rates for the decarboxylation of 5-CV by LigW were determined in $\mathrm{H}_{2} \mathrm{O}$ and $\mathrm{D}_{2} \mathrm{O}$ in $50 \mathrm{mM}$ HEPES at $\mathrm{pH}(\mathrm{D}) 7.0$, and $1.0 \mathrm{mM} \mathrm{MnCl}{ }_{2}$ at $30{ }^{\circ} \mathrm{C}$. The average of three assays that utilized $0.10 \mu \mathrm{M} \mathrm{LigW}$ in $\mathrm{D}_{2} \mathrm{O}$ and $\mathrm{H}_{2} \mathrm{O}$ were used to determine the effects on the values of $k_{\text {cat }}$ and $k_{\text {cat }} / K_{\mathrm{m}}$. The $\mathrm{pH}$ was measured using a Thermo Scientific Orion Star A214 pH/ISE Benchtop Meter with a gel filled $\mathrm{pH}$ electrode calibrated with standard buffers. A correction factor of 0.4 was added to meter $\mathrm{pH}$ reading in $\mathrm{D}_{2} \mathrm{O} .^{42}$

Deuterium Exchange Experiments. The enzyme-catalyzed exchange of the hydrogen at $\mathrm{C5}$ of the product vanillate with deuterium from solvent was measured as a function of $\mathrm{pH}$. Vanillate $(1.9 \mathrm{mM})$ was incubated in $95 \% \mathrm{D}_{2} \mathrm{O}$ at $\mathrm{pD} 9.0\left(19 \mathrm{mM} \mathrm{NH}_{4} \mathrm{HCO}_{3}\right)$ for up to 7 days at $25{ }^{\circ} \mathrm{C}$ after the addition of $48 \mu \mathrm{M} \mathrm{LigW}$. The reactions were quenched by the removal of enzyme with a $10 \mathrm{kDa}$ molecular weight cutoff filter and the solvent removed by rotary evaporation. The dried samples were reconstituted with $99 \% \mathrm{D}_{2} \mathrm{O}$ containing $1.2 \mathrm{mM}$ EDTA to coordinate any paramagnetic metal ions. The fractional exchange of deuterium for hydrogen at C5 of vanillate was determined by ${ }^{1} \mathrm{H}$ NMR spectroscopy. Similarly, the exchange of the hydrogen at $\mathrm{C5}$ of 3-methoxybenzoic acid $(1.9 \mathrm{mM})$ with deuterium from solvent was measured at $\mathrm{pD} 9.0$ in the presence of 48 $\mu \mathrm{M} \mathrm{LigW}$. The apparent first-order rate constants for the exchange of deuterium from solvent at $\mathrm{C}-5$ of vanillate were obtained from a fit of the data to eq 6 , where $A_{t} / A_{0}$ is the ratio of vanillate that is protonated at time $t$ and $k_{\text {obs }}$ is the apparent first-order rate constant for the exchange reaction. Control reactions were conducted for both vanillate and 3-methoxybenzoic acid in the absence of added enzyme.

$$
\ln \left[A_{t} / A_{0}\right]=-k_{\text {obs }} t
$$

Product Isotope Effect. The product isotope effect (PIE) was measured in a 50:50 mixture of $\mathrm{H}_{2} \mathrm{O}$ and $\mathrm{D}_{2} \mathrm{O}$ after the addition of 65 $\mu \mathrm{M} \mathrm{LigW} \mathrm{to} \mathrm{a} \mathrm{solution} \mathrm{containing} 2.0 \mathrm{mM} \mathrm{5-CV}$ at $\mathrm{pH} / \mathrm{pD}$ values of $7.0\left(20 \mathrm{mM}\right.$ potassium phosphate) and $9.0\left(20 \mathrm{mM} \mathrm{NH}_{4} \mathrm{HCO}_{3}\right)$. The reactions were allowed to continue for $1 \mathrm{~h}$, the enzyme was removed by filtration and then the solvent removed by evaporation. The fraction of hydrogen and deuterium at $\mathrm{C} 5$ was determined by integration of the NMR signal for the hydrogen at C5 relative to the nonexchangeable hydrogens at $\mathrm{C} 2$ and $\mathrm{C} 6$ of vanillate.

\section{ASSOCIATED CONTENT}

\section{S Supporting Information}

The Supporting Information is available free of charge on the ACS Publications website at DOI: 10.1021/jacs.5b08251.

Tables S1-S5, Figures S1-S8. (PDF)

Structure determination of 5-carboxyvanillate. (CIF)

Structure determination of 5-nitrovanillate. (CIF)

\section{AUTHOR INFORMATION}

\section{Corresponding Authors}

*steve.almo@einstein.yu.edu

*raushel@tamu.edu

\section{Notes}

The authors declare no competing financial interest.

\section{ACKNOWLEDGMENTS}

This work was supported in part by the NIH (GM 71790) and the Robert A. Welch Foundation (A-840). Use of the National Synchrotron Light Source, Brookhaven National Laboratory, was supported by the U.S. Department of Energy.

\section{REFERENCES}

(1) Sainsbury, P. D.; Hardiman, E. M.; Ahmad, M.; Otani, H.; Seghezzi, N.; Eltis, L. D.; Bugg, T. D. H. ACS Chem. Biol. 2013, 8, 2151-2156.
(2) Bugg, T. D. H.; Ahmad, M.; Hardiman, E. M.; Rahmanpour, R Nat. Prod. Rep. 2011, 28, 1883-1896.

(3) Tobimatsu, Y.; Chen, F.; Nakashima, J.; Escamilla-Treviño, L. L.; Jackson, L.; Dixon, R. A.; Ralph, J. Plant Cell 2013, 25, 2587-2600.

(4) Masai, E.; Katayama, Y.; Fukuda, M. Biosci., Biotechnol., Biochem. 2007, 71, 1-15.

(5) Seibert, C. M.; Raushel, F. M. Biochemistry 2005, 44, 6383-6391.

(6) Holm, L.; Sander, C. Proteins: Struct., Funct., Genet. 1997, 28, 7282.

(7) Toth, K.; Amyes, T. L.; Wood, B. M.; Chan, K.; Gerlt, J. A.; Richard, J. P. J. Am. Chem. Soc. 2010, 132, 7018-7024.

(8) Amyes, T. L.; Wood, B. M.; Chan, K.; Gerlt, J. A.; Richard, J. P. J. Am. Chem. Soc. 2008, 130, 1574-1575.

(9) Funihashi, M.; Ishida, T.; Kuroda, S.; Kotra, L. P.; Pai, E. F.; Miki, K. J. J. Am. Chem. Soc. 2013, 135, 17432-17443.

(10) Gato, M.; Hayashi, H.; Miyahara, I.; Hirotsu, K.; Yoshida, M.; Oikawa, T. J. Biol. Chem. 2006, 281, 34365-34373.

(11) Xu, S.; Li, W.; Zhu, J.; Wang, R.; Li, Z.; Xu, G.; Ding, J. Cell Res. 2013, 23, 1296-1309.

(12) Martynowski, D.; Eyobo, Y.; Li, T.; Yang, K.; Liu, A.; Zhang, H. Biochemistry 2006, 45, 10412-10421.

(13) Kluger, R.; Howe, G. W.; Mundle, S. O. C. Adv. Phys. Org. Chem. 2013, 47, 85-128.

(14) Moral, M. E.G.; Tu, C.; Richards, N. G. J.; Silverman, D. N. Anal. Biochem. 2011, 418, 73-77.

(15) Radzicka, A.; Wolfenden, R. Science 1995, 267, 90-93.

(16) Neumann, P.; Tittmann, K. Curr. Opin. Struct. Biol. 2014, 29, 122-133.

(17) Petsko, G. A.; Ringe, D. Curr. Opin. Chem. Biol. 2000, 4, 89-94.

(18) Vocadlo, D. J.; Davies, G. J.; Laine, R.; Withers, S. G. Nature 2001, 412, 835-838.

(19) Bacik, J. P.; Whitworth, G. E.; Stubbs, K. A.; Vocadlo, D. J.; Mark, B. L. Chem. Biol. 2012, 19, 1471-1482.

(20) Asztalos, P.; Parthier, C.; Golbik, R.; Kleinschmidt, M.; Hübner, G.; Weiss, M. S.; Friedemann, R.; Wille, G.; Tittmann, K. Biochemistry 2007, 46, 12037-12052.

(21) Ludtke, S.; Neumann, P.; Erixon, K. M.; Leeper, F. J.; Kluger, R.; Ficner, R.; Tittman, K. Nat. Chem. 2013, 5, 762-767.

(22) Lehwess-Litzmann, A.; Neumann, P.; Pathier, C.; Ludtke, S.; Golbik, R.; Ricner, R.; Tittmann, K. Nat. Chem. Biol. 2011, 7, 678684.

(23) Milic, D.; Demidkina, T. V.; Faleev, N. G.; Phillips, R. S.; Matkovic-Calogovic, D.; Anstson, A. A. J. Am. Chem. Soc. 2011, 133, 16468-16476.

(24) Kiss, L. E.; Ferreira, H. S.; Torrao, L.; Bonifácio, M. J.; Palma, P. N.; Soares-da-Silva, P.; Learmonth, D. A. J. Med. Chem. 2010, 53, 3396-3411.

(25) Otwinowski, Z.; Minor, W. In Methods in Enzymology; Carter, C. W. J., Sweet, R. M., Abelson, J. N., Simon, M. I., Eds.; Academic Press: New York, 1997; pp 307-326.

(26) Long, F.; Vagin, A.; Young, P.; Murshudov, G. N. Acta Crystallogr., Sect. D: Biol. Crystallogr. 2008, D64, 125-132.

(27) Emsley, P.; Cowtan, K. Acta Crystallogr., Sect. D: Biol. Crystallogr. 2004, D60, 2126-2132.

(28) Adams, P. D.; Afonine, P. V.; Bunkoczi, G.; Chen, V. B.; Davis, I. W.; Echols, N.; Headd, J. J.; Hung, L. W.; Kapral, G. J.; GrosseKunstleve, R. W.; McCoy, A. J.; Moriarty, N. W.; Oeffner, R.; Read, R. J.; Richardson, J. S.; Terwilliger, T. C.; Zwart, P. H. Acta Crystallogr., Sect. D: Biol. Crystallogr. 2011, D66, 213-221.

(29) Lamzin, V. S.; Wilson, K. S. Acta Crystallogr., Sect. D: Biol. Crystallogr. 1993, D49, 129-147.

(30) Hooft, R. W.; Vriend, G.; Sander, C.; Abola, F. Nature 1996, 381, 272-273.

(31) Chen, V. B.; Arrendall, W. B.; Headd, J. J.; Keedy, D. A.; Immormino, R. M.; Kapral, G. J.; Murray, L. W.; Richardson, J. S.; Richardson, D. C. Acta Crystallogr., Sect. D: Biol. Crystallogr. 2010, D66, 12-21. 
(32) Collaborative Computational Project Number 4 The CCP4 Suit: Programs for Protein Crystallography. Acta Crystallogr., Sect. D: Biol. Crystallogr. 1994, 50, 760-763.

(33) Delano, W. L. The PyMOL Molecular Graphics System; Delano Scientific: San Carlos, CA, 2002.

(34) Murshudov, G. N.; Vagin, A. A.; Dodson, E. J. Acta Crystallogr., Sect. D: Biol. Crystallogr. 1997, D53, 240-255.

(35) Kabsch, W. Acta Crystallogr., Sect. D: Biol. Crystallogr. 2010, D66, 125-132.

(36) Bruker APEX2, SADABS, XPREP and SAINT-Plus; Bruker AXS Inc.: Madison, WI, 2004. WinGX.

(37) Sheldrick, G. M. Acta Crystallogr., Sect. A: Found. Crystallogr. 2008, A64, 112-122.

(38) Farrugia, L. J. J. Appl. Crystallogr. 2012, 45, 849-854.

(39) Macrae, C. F.; Bruno, I. J.; Chisholm, J. A.; Edgington, P. R.; McCabe, P.; Pidcock, E.; Rodriquez-Monge, L.; Taylor, R.; van de Streek, J.; Wood, P. A. J. Appl. Crystallogr. 2008, 41, 466-470.

(40) Williams, J. W.; Morrison, J. F. Methods Enzymol. 1979, 63, 437-467.

(41) Cook, P. F.; Cleland, W. W. In Enzyme Kinetics and Mechanism; Garland Science, 2007; p 203.

(42) Krężel, A.; Bal, W. J. Inorg. Biochem. 2004, 98, 161-166. 